\title{
Bullous Eruption Caused by an Exotic Hedgehog Purchased as a Household Pet
}

\author{
Travis Frantz, BS; Robert Rampton, DO; Wendi Wohltmann, MD
}

\section{PRACTICE POINTS}

- Bullous tinea may present with little or no scale, which can lead to confusion with acute contact dermatitis.

- The recent popularity of exotic pets may increase the incidence of fungal zoonotic dermatitis.

- Prompt recognition of tinea incognito is essential when treating lesions with corticosteroids.

- Skin lesions not responding appropriately to therapy warrant reassessment and further evaluation.
An unusual case of a 37-year-old woman with bullous tinea manuum caused by an infection with Trichophyton mentagrophytes is described. The patient presented with a pruritic erythematous vesicular rash on the right palm and interdigital web spaces between the first and second digits. The preliminary diagnosis was acute contact dermatitis, which was treated with oral and topical corticosteroids. The rash failed to respond to treatment, resulting in tinea incognito. At 2-week follow-up, a potassium hydroxide $(\mathrm{KOH})$ preparation performed in clinic was positive for $T$ mentagrophytes. The suspected source of infection was a domestic 4-toed, white-bellied African pygmy hedgehog (Atelerix albiventris). There was remarkable improvement of the lesion after 2 weeks with appropriate topical and systemic antifungal agents. This case illustrates that popular exotic pets such as the hedgehog can increase the risk for zoophilic dermatophytic infection, which can present as bullous lesions with little or no scale and can be confused with acute contact dermatitis. It is important for the clinician to recognize this presentation and provide close follow-up for lesions not responding to treatment.

Cutis. 2020;105:314-316.

\section{Case Report}

A 37-year-old woman presented to the dermatology clinic with an itchy rash involving the right hand. The rash had been present for 10 days but had become increasingly pruritic and vesicular over the last 5 days. She denied new exposures or other household members with similar symptoms. The patient reported that she had purchased a 4-toed, white-bellied African pygmy hedgehog (Atelerix albiventris) approximately 4 months prior. Upon questioning, she stated that she handled the hedgehog a couple of times a week and always washed her hands with soap and water immediately after. The patient's medical and personal history were otherwise unremarkable.

Review of systems, including fevers, chills, and night sweats, was negative. Clinical examination revealed erythema with overlying vesicles and pustules on the right radial palm, radial dorsal hand, and interdigital web space of the first and second digit (Figure 1). The eruption was actively discharging serous exudate. No other lesions were present.

Unspecified acute contact dermatitis was the preliminary diagnosis based on clinical presentation and history. Other entities considered before making the diagnosis included psoriasis, eczema, and an infectious cause. Specimens were taken for bacterial and fungal cultures as well as a specimen for herpes simplex virus by polymerase chain reaction. Due to the intense pruritus and vesicular nature of the rash, the patient was treated with a 2-week, 60-40-20 prednisone taper and clobetasol propionate ointment $0.05 \%$ twice daily.

Mr. Frantz is from the Center for Dermatology Research, Department of Dermatology, Wake Forest School of Medicine, Winston-Salem, North Carolina. Dr. Rampton is from the Department of Dermatology, Tripler Army Medical Center, Honolulu, Hawaii. Dr. Wohltmann is from the Department of Dermatology, San Antonio Uniformed Services Health Education Consortium, Texas.

The authors report no conflict of interest.

The views expressed in this manuscript are those of the authors and do not reflect the official policy or position of the Department of the Army, Department of the Air Force, Department of Defense, or the US Government.

Correspondence: Travis Frantz, BS, Department of Dermatology, Wake Forest School of Medicine, 4618 Country Club Rd, Winston-Salem, NC 27104 (travisfrantz@gmail.com). 
At 1-week follow-up, the eruption had improved, but the patient was still experiencing mild pruritus. Physical examination of the affected areas showed erythematous, violaceous, annular patches with slight scale at the periphery; all bullous lesions had resolved (Figure 2). Bacterial culture and herpes simplex virus by polymerase chain reaction were negative.

Two weeks after initial consultation, the fungal culture returned positive and showed growth of Trichophyton mentagrophytes. The patient was contacted and returned for re-evaluation. Physical examination showed decreased erythema and no bullous lesions; however, there was increased fine scale throughout the affected area on the right palm and first and second interdigital spaces (Figure 3). She reported mild pruritus. A confirmatory potassium hydroxide $(\mathrm{KOH})$ preparation was positive for fungal hyphae. The patient was subsequently

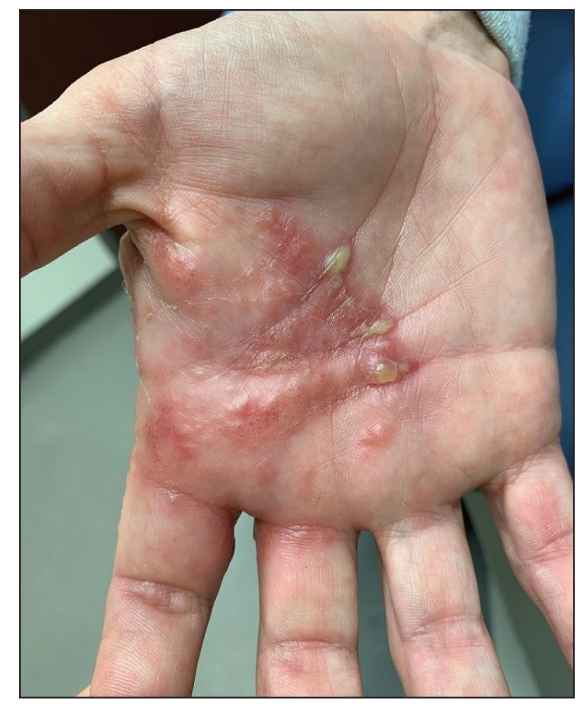

FIGURE 1. Erythematous vesiculobullous plaque on the right palm, interdigital web spaces, and radial aspect of first digit, with pustules and actively discharging serous exudate.

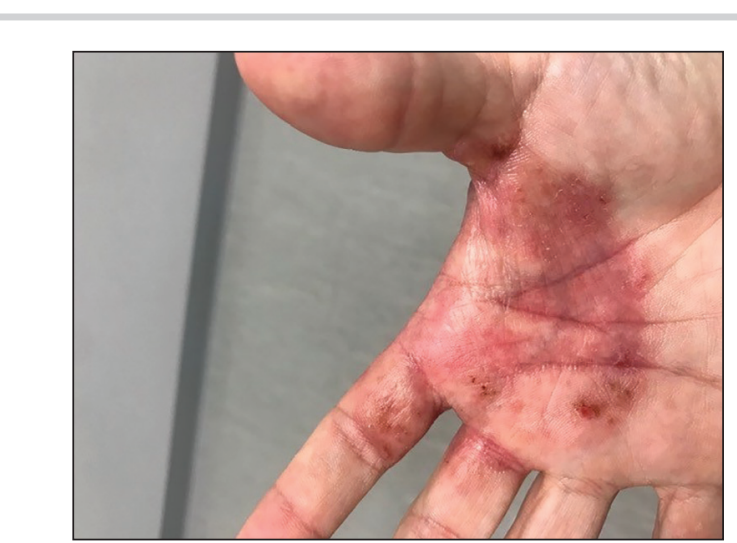

FIGURE 2. After 1 week of therapy with prednisone and clobetasol propionate ointment $0.05 \%$, the eruption had improved, but there was an annular violaceous patch on the right radial palm with a few erosions at the periphery of the lesion. diagnosed with bullous tinea secondary to domestic hedgehog exposure that was now presenting as tinea manuum incognita. After 2 weeks of appropriate systemic and topical antifungal therapy, the patient's skin eruption markedly improved (Figure 4).

\section{Comment}

Tinea manuum is a dermatophytic epidermal infection of the hand. The most common causative organisms are Trichophyton rubrum, $T$ mentagrophytes, and Epidermophyton floccosum. Infection can be acquired from contact with an infected person or animal, fomites, soil, or autoinoculation. Tinea manuum often is associated with tinea pedis. The hand that is used to excoriate the pruritic feet becomes infected, resulting in the classic two feet-one hand syndrome, which this patient did not have. ${ }^{1}$

Dermatophytes colonize keratin-containing tissuesskin, hair, and nails-utilizing the keratin for nutrients,

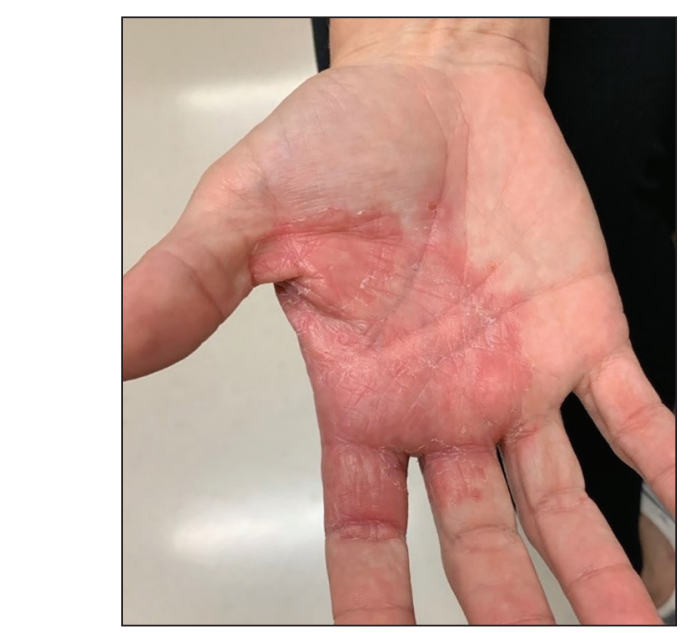

FIGURE 3. After 2 weeks of therapy, there was an erythematous patch with overlying fine scale.

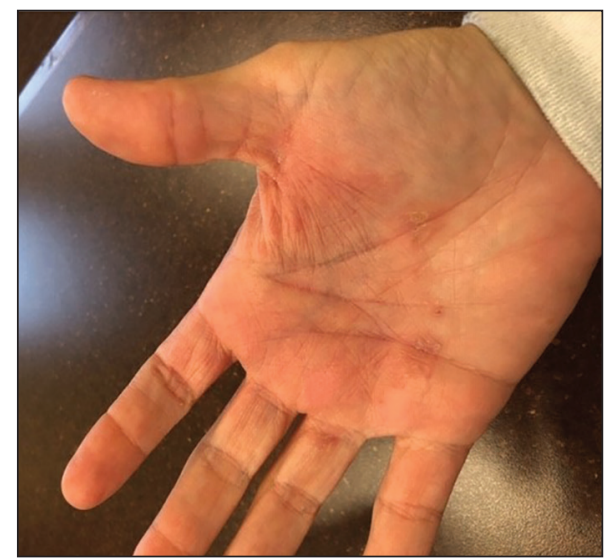

FIGURE 4. After 2 weeks of systemic and topical antifungal therapy (4 weeks after initial presentation), the fungal infection was resolving, with decreased scale, erosions, and annular configuration. 


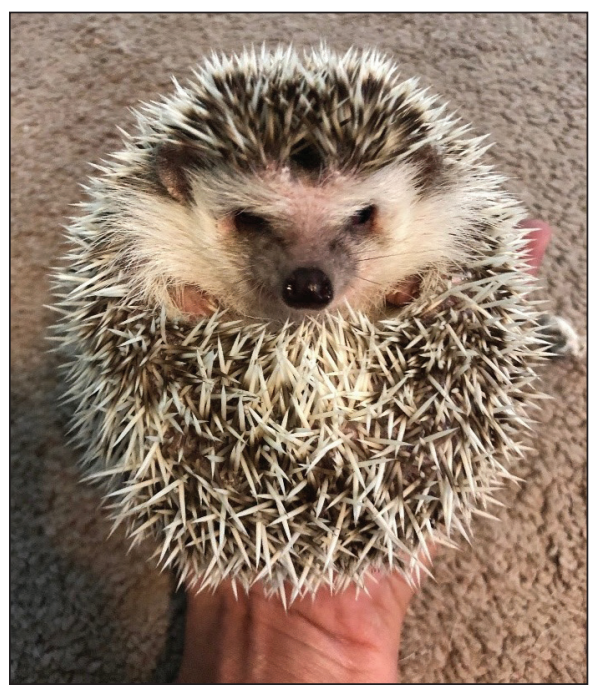

FIGURE 5. The patient's 4-toed, white-bellied African pygmy hedgehog (Atelerix albiventris) in a curled position.

and they do not invade living tissue in immunocompetent hosts. Dermatophytes cause clinical disease from an allergic host response to fungal antigens or their metabolic products. ${ }^{1}$ Tinea incognito results from the use of corticosteroids to treat a cutaneous fungal infection. The immunomodulatory effects of corticosteroids alter the appearance of the lesion. Hallmark signs and symptoms of a tinea infection, including scale, prominent border, erythema, and pruritus, can be reduced with corticosteroid use, giving the false impression that the lesion is resolving. ${ }^{2,3}$

The diagnosis of tinea manuum can be made clinically and often is supported with the findings of a $\mathrm{KOH}$ preparation. Scraping from an active scaling border generally provides the best results for obtaining fungal elements. For vesiculobullous lesions, the roof of a vesicle can provide an adequate specimen. Fungal culture and specific dermatophyte testing mediums can be used as confirmatory tests or allow for speciation, which help establish the diagnosis. ${ }^{1}$

Trichophyton mentagrophytes is a species complexa group of closely related organisms that share morphologic appearance to the point that boundaries between them often are unclear. It can be identified by gross and microscopic morphology; however, variants of $T$ mentagrophytes (eg, Trichophyton interdigitale, Trichophyton erinacei) require a confirmatory test or molecular analysis to be correctly identified. ${ }^{4-6}$ The laboratory used at our facility does not routinely attempt to identify the variant due to of lack of clinical significance. ${ }^{7,8}$

Anthropophilic fungi such as T rubrum, E floccosum, and $T$ interdigitale generally do not cause a robust immunologic reaction. Infection usually is chronic in nature, though cases of pustular and vesicular tinea have been described. ${ }^{9,10}$ Trichophyton erinacei and $T$ mentagrophytes are zoophilic dermatophytes that cause an acute host response and are more likely to present with vesiculobullous lesions. Trichophyton erinacei is the most common fungal pathogen associated with $A$ albiventris and has been isolated from its epidermal mites and quills, ${ }^{11,12}$ which likely facilitates interspecies transmission and compromises the cutaneous barrier of human hosts when the hedgehog is handled.

Atelerix albiventris is the most common domesticated hedgehog in the United States. These mild-mannered, nocturnal insectivores are unique, low-maintenance pets that have recently gained popularity. They are notable for their propensity to curl into a ball when frightened (Figure 5). The spines are not barbed and do not detach, as those of a porcupine do, but are still capable of piercing the skin. Atelerix albiventris is known to cause zoonotic dermatosis in humans and should be handled with gloves. ${ }^{13}$ Performing a KOH preparation early in the diagnostic workup can help initiate antifungal therapy, as results of fungal culture can take several weeks.

\section{Conclusion}

This case illustrates the importance of close follow-up of skin lesions that only partially respond to initial treatment and maintaining a high index of suspicion as exotic pets become popular.

\section{REFERENCES}

1. Elewski BE, Hughey LC, Hunt KM, et al. Fungal diseases. In: Bolognia JL, Schaffer JV, Cerroni L, eds. Dermatology. 4th ed. Philadelphia, PA: Elsevier; 2018:1329-1363.

2. Habif T. Superficial fungal infections. In: Habif T. Clinical Dermatology. 6th ed. Philadelphia, PA: Elsevier; 2016:487-533.

3. Lange M, Jasiel-Walikowska E, Nowicki R, et al. Tinea incognito due to Trichophyton mentagrophytes. Mycoses. 2010;53:455-457.

4. Pchelin IM, Azarov DV, Churina MA, et al. Species boundaries in the Trichophyton mentagrophytes/T. interdigitale species complex. Med Mycol. 2019;57:781-789.

5. Makimura K, Mochizuki T, Hasegawa A, et al. Phylogenetic classification of Trichophyton mentagrophytes complex strains based on DNA sequences of nuclear ribosomal internal transcribed spacer 1 regions. J Clin Microbiol. 1998;36:2629-2633.

6. de Hoog GS, Dukik K, Monod M, et al. Toward a novel multilocus phylogenetic taxonomy for the dermatophytes. Mycopathologia. 2017;182:5-31.

7. Rudramurthy SM, Shankarnarayan SA, Dogra S, et al. Mutation in the squalene epoxidase gene of Trichophyton interdigitale and Trichophyton rubrum associated with allylamine resistance. Antimicrob Agents Chemother. 2018;62:e02522-17.

8. Singh A, Masih A, Khurana A, et al. High terbinafine resistance in Trichophyton interdigitale isolates in Delhi, India harbouring mutations in the squalene epoxidase gene. Mycoses. 2018;61:477-484.

9. KawakamiY, Oyama N, Sakai E, et al. Childhood tinea incognito caused by Trichophyton mentagrophytes var. interdigitale mimicking pustular psoriasis. Pediatr Dermatol. 2011;28:738-739.

10. Neri I, Piraccini BM, Guareschi E, et al. Bullous tinea pedis in two children. Mycoses. 2004;47:475-478.

11. Abarca ML, Castellá G, Martorell J, et al. Trichophyton erinacei in pet hedgehogs in Spain: occurrence and revision of its taxonomic status. Med Mycol. 2016;55:164-172.

12. Morris P, English MP. Transmission and course of Trichophyton erinacei infections in British hedgehogs. Sabouraudia. 1973;11:42-47.

13. Riley PY, Chomel BB. Hedgehog zoonoses. Emerg Infect Dis. 2005;11:1-5. 